\title{
Pseudopotential hyperfine calculations through perturbative core-level polarization
}

\author{
Mohammad Saeed Bahramy, ${ }^{1, *}$ Marcel H. F. Sluiter, ${ }^{2}$ and Yoshiyuki Kawazoe ${ }^{1}$ \\ ${ }^{1}$ Institute for Materials Research, Tohoku University, Sendai 980-8577, Japan \\ ${ }^{2}$ Department of Materials Science and Engineering, Delft University of Technology, Mekelweg 2, 2628CD Delft, The Netherlands
}

(Received 23 November 2006; revised manuscript received 12 February 2007; published 30 July 2007)

\begin{abstract}
Within density functional theory, an efficient and accurate method for calculating the hyperfine parameters in the context of pseudopotential formalism is proposed. The spin density at and in the vicinity of the nucleus is evaluated in two steps. First, a transformation due to Blöchl [Phys. Rev. B 50, 17953 (1994)] is applied to reconstruct the frozen-core all-electron wave functions in the core regions. Second, the contributions of core orbitals to the charge density at the nucleus are evaluated through first-order perturbation theory in which the perturbing potential is defined as a functional of charge and spin densities. The current pseudopotential based method makes it possible to predict hyperfine parameters of complex molecular assemblies and crystal defects with an accuracy as good as current all-electron method with less computational cost.
\end{abstract}

DOI: 10.1103/PhysRevB.76.035124

PACS number(s): 71.15.-m, 31.15.Ew, 31.30.Gs, 32.10.Fn

\section{INTRODUCTION}

Much of our knowledge on the geometrical and electronic structure of molecules and clusters as well as the pointlike defects in crystals has been obtained through experimental techniques such as electron paramagnetic resonance and nuclear magnetic resonance. ${ }^{1-3}$ Common to all these techniques is the measurement of the so-called hyperfine structure, which describes the interaction of the electronic wave functions with the nuclear magnetic moments. In general, the hyperfine structure is decomposed into the isotropic (Fermicontact) parameter $A_{i s o}$ and anisotropic (dipolar) parameter $A_{\text {aniso }}$. The former is directly proportional to the spin density at the nucleus, arising from the spin-polarized $s$-like orbitals, while the latter has contributions from the other spinpolarized states, e.g., $p$ - or $d$-like orbitals, and is proportional to the volume integral over the spin density divided by the distance to the nucleus to the third power. In actuality, molecular motion causes $A_{\text {aniso }}$ to average out to zero so that only $A_{\text {iso }}$ can be observed in the spectra of gases and liquids.

Although the theory underlying the hyperfine structure is well understood and was already developed in the early days of quantum mechanics, ${ }^{1,2}$ the first-principles calculations of hyperfine parameters (HFPs) have proven to be a challenging task. ${ }^{4,5}$ The main challenge is the necessity of an accurate representation of the spin density at and in the vicinity of the nucleus. Already a half century ago, works based on the unrestricted Hartree-Fock method ${ }^{6,7}$ revealed that the spinpolarized core states contribute substantially to the spin density at the nuclei of system with non-s-like singly occupied molecular orbitals (SOMOs). ${ }^{8-10}$ Therefore, contributions of both core and valence states in the vicinity of the nucleus must be accurately accounted for.

Among the computational approaches, all-electron (AE) methods based on density functional theory (DFT), ${ }^{11}$ such as the linearized muffin-tin orbital, ${ }^{12}$ the full-potential linearized augmented-plane-wave, ${ }^{13}$ and the all-electron mixedbasis $(\mathrm{AEMB})^{14}$ methods have proven to give accurate representations of the spin density near the nucleus. However, $\mathrm{AE}$ methods are computationally less efficient than planewave pseudopotential (PP) methods. Additionally, new de- velopments in DFT are typically more easily coded within PP methods than in AE methods. Therefore, already some time ago it was realized that it is desirable to be able to compute HFPs with PP methods. ${ }^{15}$ However, because of the inherent shortcoming of PP methods in approximating the electronic wave functions within the core region, PP methods cannot be used in their original form for the calculation of HFPs. The difficulties originate from (1) the nodeless behavior of pseudo-wave-functions in the core region, resulting in incorrect spin densities in the vicinity of the nucleus, and (2) the complete elimination of core orbitals and, consequently, the complete elimination of the core spin polarization and corresponding contributions to the HFPs.

So far, there have been several efforts in developing a pseudopotential treatment for the calculation of HFPs. However, most of these efforts have been concentrated on the first challenge, that is, the reconstruction of the full-nodal form of AE wave functions. ${ }^{16-18}$ In this regard, the so-called projector-augmented-wave (PAW) method proposed by Blöchl ${ }^{19}$ has shown to yield highly accurate frozen-core wave functions in the core regions and hence is considered as a reliable method for calculating HFPs of systems with $s$-like SOMO, e.g., hydrogen defects in $\mathrm{Si}^{15}$ Another important aspect of HFPs computed through PP formalism has only very recently received attention: the effect of core spin polarization was considered by Yazyev et al. ${ }^{20}$ and Declerck et $a l .{ }^{21}$ The former ${ }^{20}$ is similar in spirit to the current work, but approaches the treatment of the core spin polarization differently. In the so-called core spin-polarization correction (CSPC) method, ${ }^{20}$ a reconstruction of the $\mathrm{AE}$ wave functions and the frozen-valence spin-density approximation are used to solve the Kohn-Sham equations for core states only. Despite the simplicity of the method, it seems to be quite accurate for calculating $A_{i s o}$ of simple molecular radicals, containing main group atoms. However, the validity of the method has not been further examined for more complicated structures including heavier species, i.e., transition metal atoms. Moreover, within this approach, still one cannot calculate the anisotropic HFPs. In the latter, ${ }^{21}$ a hybrid method is proposed in which the nuclei of interest are described with an all-electron treatment and a PP approximation is used for 
the remaining atoms. Nontheless, this method appears sensitive to the choice of basis set.

Here, we present a general and accurate method for calculating HFPs, within the DFT pseudopotential formalism. The approach, which we call perturbative core-level polarization (PCLP), is based on the evaluation of HFPs by taking into account the contributions of both core and valence spinpolarized orbitals to the spin density at and in the vicinity of the nucleus. In this regard, we first reconstruct the $\mathrm{AE}$ wave functions from the corresponding pseudo (PS)-wavefunctions with the assumption that core electrons are unperturbed by the presence of other atoms, also known as the frozen-core approximation. Then, the reconstructed AE wave functions are utilized to develop a local spin-density functional potential. Finally, using this perturbing potential, the spin-polarized core levels are estimated by means of firstorder perturbation theory.

It is worth mentioning that other perturbation treatments of core spin polarization for the computation of isotropic HFPs have been derived within the context of Hartree-Fock (HF) formalism. ${ }^{22,23}$ These studies are mainly concentrated on simple atomic and molecular models. This is understandable because of the inherent neglect of electron-electron correlation effects and the leading spin contamination errors in HF approximations make their utilization a nontrivial task for larger systems with, e.g., $d$-type atoms. In contrast, in the current DFT-based approach, these problems are essentially negligible and of little importance.

\section{FORMULATION}

The hyperfine parameters $A_{\text {iso }}$ and $A_{\text {aniso }}$ are defined as

$$
A_{\text {iso }}(I)=\frac{2}{3} \mu_{0} g_{e} \mu_{e} g_{I} \mu_{I} \rho_{s}\left(\mathbf{R}_{I}\right)
$$

and

$$
A_{\text {aniso }}(I)=\frac{1}{4 \pi} \mu_{0} g_{e} \mu_{e} g_{I} \mu_{I} \int d \mathbf{r}_{I} \rho_{s}\left(\mathbf{r}_{I}\right) \frac{3 \cos ^{2} \theta-1}{2 r_{I}^{3}},
$$

where $\mathbf{R}_{I}$ is the position of nucleus $I, \mathbf{r}_{I}$ is the positional vector relative to the nucleus $\left(\mathbf{r}_{I}=\mathbf{r}-\mathbf{R}_{I}\right), \theta$ is the angle between $\mathbf{r}_{I}$ and symmetry axes, $\mu_{0}$ is the permeability of vacuum $\left(4 \pi \times 10^{-7} \mathrm{~T}^{2} \mathrm{~m}^{3} \mathrm{~J}^{-1}\right), g_{e}$ is the electron $g$ factor, $\mu_{e}$ is the Bohr magneton, and $g_{I}$ and $\mu_{I}$ are the gyromagnetic ratio and the magnetic moment of the nucleus. Throughout this work, $g_{I}$ and $\mu_{I}$ values are taken from Ref. 24. The spin density $\rho_{s}(\mathbf{r})$ is the difference between the spin-up and spindown charge densities, $\rho_{s}(\mathbf{r})=\rho^{\uparrow}(\mathbf{r})-\rho^{\downarrow}(\mathbf{r})$. Using the notation $\sigma$ for spin sign ( $\uparrow$ or $\downarrow), \rho^{\sigma}(\mathbf{r})$ can be considered as a sum of the core contribution $\rho^{c \sigma}(\mathbf{r})$ and the valence contribution $\rho^{v \sigma}(\mathbf{r})$,

$$
\rho^{\sigma}(\mathbf{r})=\rho^{c \sigma}(\mathbf{r})+\rho^{v \sigma}(\mathbf{r}) .
$$

In the conventional PP formalism, the core contribution $\rho^{c \sigma}(\mathbf{r})$ is not available. Additionally, the valence contribution is expressed as

$$
\tilde{\rho}^{v \sigma}(\mathbf{r})=\sum_{n}\left\langle\tilde{\Psi}_{n}^{\sigma} \mid \mathbf{r}\right\rangle\left\langle\mathbf{r} \mid \widetilde{\Psi}_{n}^{\sigma}\right\rangle,
$$

where the PS-wave-functions $\left|\widetilde{\Psi}_{n}^{\sigma}\right\rangle$ differ from the exact $\mathrm{AE}$ wave functions $\left|\Psi_{n}^{\sigma}\right\rangle$ within the core regions. Thus, any attempt to implement HFP calculations into PP methods must consider (1) the inclusion of $\rho^{c \sigma}$ in the charge density and (2) the reconstruction of $\left|\Psi_{n}^{\sigma}\right\rangle$ from $\left|\widetilde{\Psi}_{n}^{\sigma}\right\rangle$ inside the atomic spheres.

Below, we first discuss the second task. The $\left|\Psi_{n}^{\sigma}\right\rangle$ is reconstructed from $\left|\widetilde{\Psi}_{n}^{\sigma}\right\rangle$ using the PAW ${ }^{19}$ method. In this context, $\left|\Psi_{n}^{\sigma}\right\rangle$ are obtained by applying the following transformation on $\left|\tilde{\Psi}_{n}^{\sigma}\right\rangle$ :

$$
\left|\Psi_{n}^{\sigma}\right\rangle=\left|\tilde{\Psi}_{n}^{\sigma}\right\rangle+\sum_{\lambda}\left(\left|\phi_{\lambda}\right\rangle-\left|\tilde{\phi}_{\lambda}\right\rangle\right)\left\langle\tilde{p_{\lambda}} \mid \tilde{\Psi}_{n}^{\sigma}\right\rangle
$$

where AE partial waves $\left|\phi_{\lambda}\right\rangle$ are the solution of the radial Schrödinger equation and PS-partial-waves $\left|\widetilde{\phi_{\lambda}}\right\rangle$ are smooth functions that coincide with the corresponding AE partial waves outside the cutoff radius $R_{c}$. The index $\lambda$ is a shorthand denoting the particular angular momenta $l$ and $m$ of level $\lambda$, localized at the atomic site $\mathbf{R}_{I}$. The functions $\left|\tilde{p_{\lambda}}\right\rangle$ are projector functions which are well localized inside the core region and defined such that they fulfill the condition

$$
\left\langle\tilde{p_{\lambda}} \mid \tilde{\phi}_{\kappa}\right\rangle=\delta_{\lambda \kappa} .
$$

To determine $\left|\tilde{p_{\lambda}}\right\rangle$, we follow the approach proposed by Hetényi et al. ${ }^{18}$ The procedure is briefly discussed below. Initially, we perform a simple $\mathrm{AE}$ calculation (e.g., using the Herman-Skillman method ${ }^{25}$ ) for an isolated atom in the ground state in order to compute the partial waves $\left|\phi_{\lambda}\right\rangle$ and the corresponding eigenvalues $\varepsilon_{\lambda}$ as well as the radial effective potential $V_{\text {eff }}(r)$,

$$
\left(-\frac{\nabla^{2}}{2}+V_{e f f}\right)\left|\phi_{\lambda}\right\rangle=\varepsilon_{\lambda}\left|\phi_{\lambda}\right\rangle .
$$

Next, an arbitrary potential $V_{l o c}(r)$ is constructed such that it behaves smoothly inside and coincides with $V_{\text {eff }}(r)$ outside the core region. A possible choice is to find an exponential function for $V_{l o c}(r)$, that joins smoothly to $V_{\text {eff }}(r)$ at $R_{c}$ (see Ref. 19). Using $V_{l o c}(r), \varepsilon_{\lambda}$, and PS-partial-waves $\left|\widetilde{\phi_{\lambda}}\right\rangle$, a set of functions, localized within the core region, is defined as

$$
\left|\tilde{\chi_{\lambda}}\right\rangle=\left(\varepsilon_{\lambda}+\frac{\nabla^{2}}{2}-V_{l o c}\right)\left|\tilde{\phi_{\lambda}}\right\rangle \text {. }
$$

Finally, taking a linear combination of $\left|\tilde{\chi_{\lambda}}\right\rangle$, the projector functions $\left|\tilde{p_{\lambda}}\right\rangle$ are constructed as

$$
\left|\tilde{p_{\lambda}}\right\rangle=\sum_{\kappa}\left(B^{-1}\right)_{\lambda \kappa}\left|\tilde{\chi_{\kappa}}\right\rangle,
$$

where the matrix elements $B_{\lambda \kappa}$ are defined so that the computed $\left|\tilde{p_{\lambda}}\right\rangle$ fulfills the orthogonality condition [Eq. (6)],

$$
B_{\lambda \kappa}=\left\langle\tilde{\phi_{\lambda}} \mid \tilde{\chi_{\kappa}}\right\rangle .
$$


Once the projector functions $\left|\tilde{p_{\lambda}}\right\rangle$ are determined, transformation (5) can be applied to obtain $\left|\Psi_{n}^{\sigma}\right\rangle$. Similarly, the valence contribution $\rho^{v \sigma}(\mathbf{r})$ is reconstructed from $\widetilde{\rho}^{v \sigma}(\mathbf{r})$ as follows: ${ }^{19}$

$$
\rho^{v \sigma}(\mathbf{r})=\widetilde{\rho}^{v \sigma}(\mathbf{r})+\rho_{1}^{\sigma}(\mathbf{r})-\widetilde{\rho}_{1}^{\sigma}(\mathbf{r}),
$$

where

$$
\rho_{1}^{\sigma}(\mathbf{r})=\sum_{n} \sum_{\lambda \kappa}\left\langle\tilde{\Psi}_{n}^{\sigma} \mid \tilde{p}_{\lambda}\right\rangle\left\langle\phi_{\lambda} \mid \mathbf{r}\right\rangle\left\langle\mathbf{r} \mid \phi_{\kappa}\right\rangle\left\langle\tilde{p}_{\kappa} \mid \tilde{\Psi}_{n}^{\sigma}\right\rangle
$$

and

$$
\tilde{\rho}_{1}^{\sigma}(\mathbf{r})=\sum_{n} \sum_{\lambda \kappa}\left\langle\tilde{\Psi}_{n}^{\sigma} \mid \tilde{p}_{\lambda}\right\rangle\left\langle\widetilde{\phi}_{\lambda} \mid \mathbf{r}\right\rangle\left\langle\mathbf{r} \mid \widetilde{\phi}_{\kappa}\right\rangle\left\langle\widetilde{p}_{\kappa} \mid \tilde{\Psi}_{n}^{\sigma}\right\rangle
$$

Having $\rho^{v \sigma}(\mathbf{r})$, the next step is to construct the core contribution $\rho^{c \sigma}(\mathbf{r})$. Recently, we proposed that $\rho^{c \sigma}(\mathbf{r})$ can be estimated using first-order perturbation theory. ${ }^{14}$ In this context, the spin-polarized core orbitals $\phi_{\lambda}^{\sigma}$ are constructed from a linear combination of non-spin-polarized eigenstates $\phi_{\lambda}$ as

$$
\left|\phi_{\lambda}^{\sigma}\right\rangle=\left|\phi_{\lambda}\right\rangle+\sigma \sum_{\kappa \neq \lambda} C_{\lambda \kappa}\left|\phi_{\kappa}\right\rangle
$$

where we have used the fact that the perturbing potential $\Delta V_{\sigma}$ differs just in sign for the spin-up and spin-down channels [see Eq. (18)] so that

$$
C_{\lambda \kappa}=\sigma \frac{\left\langle\phi_{\kappa}\left|\Delta V_{\sigma}\right| \phi_{\lambda}\right\rangle}{\varepsilon_{\lambda}-\varepsilon_{\kappa}}
$$

does not depend on $\sigma$. Equations. (14) and (15) suggest that only levels $\kappa$ need to be taken into account, which are energetically close to the given level $\lambda$ and for which $\left\langle\phi_{\kappa}\left|\Delta V_{\sigma}\right| \phi_{\lambda}\right\rangle$ is considerable. It is to be noted that both $\varepsilon_{\lambda}$ and $\phi_{\lambda}$ were calculated already for the reconstruction of the $\mathrm{AE}$ wave functions.

Using Eq. (14), the contribution of the core orbitals to the charge density is expressed as

$$
\rho^{c \sigma}(\mathbf{r})=\sum_{\lambda}^{\text {core }}\left\langle\phi_{\lambda}^{\sigma} \mid \mathbf{r}\right\rangle\left\langle\mathbf{r} \mid \phi_{\lambda}^{\sigma}\right\rangle
$$

where the summation is over the core levels only. Correspondingly, one can show that the contribution of the core levels to the spin density becomes

$$
\rho_{s}^{c}(\mathbf{r})=\rho^{c \uparrow}(\mathbf{r})-\rho^{c \downarrow}(\mathbf{r})=4 \sum_{\lambda}^{\text {core }} \operatorname{Re}\left[\left\langle\phi_{\lambda} \mid \mathbf{r}\right\rangle \sum_{\kappa \neq \lambda} C_{\lambda \kappa}\left\langle\mathbf{r} \mid \phi_{\kappa}\right\rangle\right],
$$

where "Re" indicates the real part. If the summation is only over the core $s$ levels (e.g., in the case of isotropic HFPs), the imaginary part vanishes.

Returning to Eq. (15), using the local spin-density approximation (LSDA), ${ }^{26}$ it is possible to define a density functional form for $\Delta V_{\sigma}$ [see Eq. (105) in Ref. 14],

$$
\Delta V_{\sigma} \simeq-\frac{\sigma}{\pi} \frac{\rho^{\operatorname{SOMO}}(\mathbf{r})}{\hat{\rho}^{2 / 3}(\mathbf{r})},
$$

where $\rho^{S O M O}=\rho^{v \uparrow}(\mathbf{r})-\rho^{v \downarrow}(\mathbf{r})$. The total charge density $\hat{\rho}(\mathbf{r})$, within the frozen-core approximation, is expressed as

$$
\hat{\rho}(\mathbf{r})=\rho^{v \uparrow}(\mathbf{r})+\rho^{v \downarrow}(\mathbf{r})+2 \sum_{\lambda}^{\text {core }}\left\langle\phi_{\lambda} \mid \mathbf{r}\right\rangle\left\langle\mathbf{r} \mid \phi_{\lambda}\right\rangle .
$$

In defining a DFT-based perturbing potential in Eq. (18), one may consider the various generalized gradient approximation (GGA) formulations instead of the LSDA. However, as Asada and Terakura ${ }^{27}$ have shown, GGAs produce a singularity in the exchange potential $\Delta V_{\sigma}$ at the nucleus position. A possible way to eliminate this singularity is to represent the nucleus not as a point but as a sphere with a finite radius within which the nuclear charge is homogeneously distributed. However, the work by Battocletti et al. ${ }^{28}$ has shown that the "finite nucleus model+GGA" does not produce significant improvement over LSDA within the context of hyperfine calculations.

It is to be noted that the perturbing potential, defined in Eq. (18), differs for the two spin channels in sign only. Thus, it is more convenient to replace $\Delta V_{\sigma}$ in Eq. (15) by an effective perturbing potential,

$$
\Delta V_{e f f}=\Delta V_{\uparrow}-\Delta V_{\downarrow}=-\frac{2}{\pi} \frac{\rho^{\operatorname{SOMO}}(\mathbf{r})}{\hat{\rho}^{2 / 3}(\mathbf{r})},
$$

so that it is necessary to estimate the core spin polarization in one spin channel only (here, we arbitrarily selected the spin-up channel).

Equation (20) reveals that $\Delta V_{\text {eff }}(\mathbf{r})$ depends sensitively on the type of SOMO. That is, if $s$-like orbitals contribute substantially to the SOMO, $\Delta V_{\text {eff }}(\mathbf{r})$ expands significantly outside the core region (see Figs. 3 and 12 in Ref. 14). In that case, the effect of core spin polarization is expected to be small. As a result, $s$-like SOMOs lead to a dominant isotropic HFP $A_{\text {iso }}$, whereas $A_{\text {aniso }}$ is likely to be negligible. On the other hand, if the SOMOs are dominated by $p$ - or $d$-like orbitals, $\Delta V_{\text {eff }}(\mathbf{r})$ becomes more localized inside the core region resulting in a large spin polarization among core orbitals (see Figs. 3 and 12 in Ref. 14). Then, both core and valence spin-polarized levels contribute significantly to $A_{i s o}$. However, as the SOMO is non-s-like, $A_{\text {aniso }}$ is substantially dominated by SOMO with a relatively small contribution from spin-polarized core levels. Hence, in both situations, $s$-like and non-s-like SOMOs, the neglect of core spin polarization may not affect seriously the $A_{\text {aniso }}$ values, while the neglect of core spin polarization for non-s-like SOMOs results in erroneous $A_{\text {iso }}$ values.

Figure 1 illustrates the radial part of $\Delta V_{\text {eff }}(\mathbf{r})$ obtained directly from an all-electron Kohn-Sham DFT calculation and as obtained from Eq. (18) for $\mathrm{C}$ with a $2 p$-type SOMO. It clearly shows that the perturbing potential is strongly localized inside the core region $\left(R_{c}\right.$ for carbon is about $\left.0.6 \AA\right)$. The figure also shows that $\Delta V_{\text {eff }}(\mathbf{r})$ from Eq. (18) coincides well with that obtained directly from the AE Kohn-Sham calculation inside the core region. Evidently, Eq. (18) is ad- 


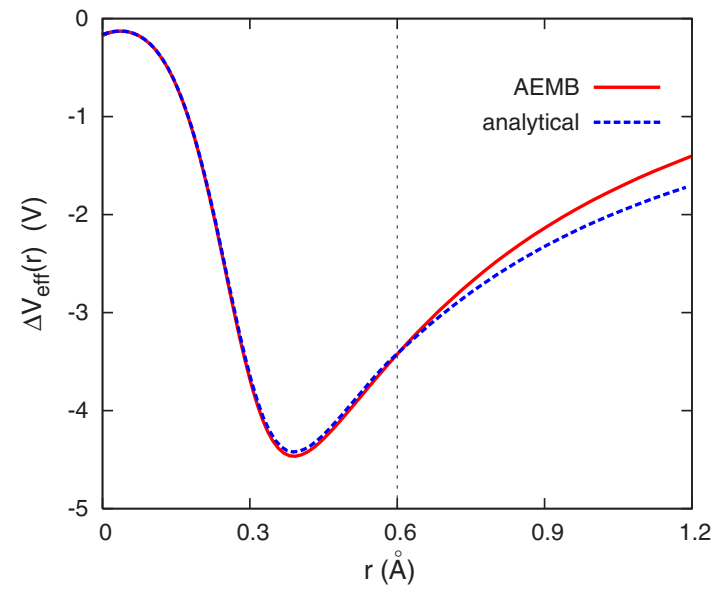

FIG. 1. (Color online) Comparison of perturbing potential $\Delta V_{\text {eff }}$ obtained directly from an all-electron mixed-basis calculation (marked with "AEMB") and as computed from Eq. (20) (marked with "analytical") for a single isolated carbon atom in its ground state configuration. The vertical dotted line indicates the cutoff radius of carbon $\left(R_{c}=0.6 \AA\right)$.

equate for estimating core spin polarization. At this stage, $A_{\text {iso }}$ and $A_{\text {aniso }}$ are calculated through Eqs. (1) and (2) by substituting the spin density $\rho^{s}(\mathbf{r})$ obtained from the charge densities, $\rho^{c \sigma}(\mathbf{r})$ and $\rho^{v \sigma}(\mathbf{r})$. In the following, the accuracy of our method is examined by calculating the HFPs of various systems and comparing the results with other computational methods and with experiment.

\section{COMPUTATIONAL DETAILS}

In this work, the DFT calculations have been carried out using the gradient-corrected PW91 ${ }^{29,30}$ exchange-correlation functional. For sake of comparison, calculations for the free atoms have been performed also using the $\mathrm{LSDA}^{31}$ functional (see Table I). For the reconstruction of AE wave functions $\left|\Psi_{n}^{\sigma}\right\rangle$, the PS-orbitals $\left|\widetilde{\phi}_{\lambda}\right\rangle$ and the PS-wave-functions $\left|\widetilde{\Psi}_{n}^{\sigma}\right\rangle$ were generated using ultrasoft pseudopotentials (USPPs), ${ }^{32}$ as implemented in the VASP code. ${ }^{33}$ Additionally, the AE orbitals $\left|\widetilde{\phi}_{\lambda}\right\rangle$ and corresponding eigenvalues $\left|\widetilde{\varepsilon}_{\lambda}\right\rangle$ were computed using the ATOMDEF package ${ }^{34}$ with gradientcorrected exchange $^{35}$ and Vosko-Wilk-Nusair correlation ${ }^{36}$ functionals. For the atomic and molecular systems, we considered a cubic supercell with a side of $16 \AA$ and integrations in reciprocal space used the $\Gamma$ point only. For the crystalline point defect calculation, a $6 \times 6 \times 6$ Monkhorst-Pack mesh has been used to sample the irreducible Brillouin zone. Stopping criterion for structural relaxations was a magnitude of the force less than $0.001 \mathrm{eV} / \AA$ on each atom.

\section{RESULTS AND DISCUSSIONS}

As a first step to evaluate the accuracy of our method, the feasibility of reconstructing $\mathrm{AE}$ wave functions via Eq. (5) is examined. Figure 2 displays the radial part of the carbon $2 s$ orbital as obtained directly from an AEMB calculation and as reconstructed from the corresponding PS-orbital by means of Eq. (5). The figure clearly shows the excellent agreement between the reconstructed $2 s$ orbital and the corresponding $\mathrm{AE}$ orbital. In fact, the lines are essentially indistinguishable.

To further test the accuracy of our method, the contribution of core levels to the spin density at the nucleus, $\rho_{s}^{c}(\mathbf{0})$, as well as the contribution of both core and valence levels to the isotropic HFP, $A_{i s o}^{c}$ and $A_{i s o}^{v}$, respectively, for various atoms, including a series of first-row elements and $3 d$ transition metals, in their ground state electronic configurations have been calculated using both $\mathrm{LSDA}^{31}$ and $\mathrm{GGA}^{29}$ functionals (see Table I).

TABLE I. Contribution of core (and valence) levels to the spin density at the nucleus, $\rho_{s}^{c}(\mathbf{0})$ (in $e / \AA^{3}$ ), and, correspondingly, to the isotropic HFP's, $A_{i s o}^{c}$ (and $A_{i s o}^{v}$ ) (in MHz), as computed using the perturbative core-level polarization (PCLP) method and as directly obtained from an all-electron mixed-basis (AEMB) calculation, for a series of first-row elements and $3 d$ transition metals. The spin configurations all follow Hund's rule.

\begin{tabular}{|c|c|c|c|c|c|c|c|c|c|c|}
\hline \multirow[b]{2}{*}{ Atom } & \multirow[b]{2}{*}{ Configuration } & \multicolumn{3}{|c|}{$\rho_{s}^{c}(\mathbf{0})$} & \multicolumn{3}{|c|}{$A_{i s o}^{c}$} & \multicolumn{3}{|c|}{$A_{i s o}^{v}$} \\
\hline & & PCLPa & PCLP ${ }^{b}$ & AEMB & PCLPa & PCLP & AEMB & PCLP $^{\mathrm{a}}$ & PCLP & AEMB \\
\hline $\mathrm{C}$ & $2 s^{2} 2 p^{2}\{2 \uparrow, 0 \downarrow\}$ & -1.344 & -1.350 & -1.351 & -111.9 & -112.4 & -112.4 & 130.2 & 132.4 & 133.8 \\
\hline $\mathrm{N}$ & $2 s^{2} 2 p^{3}\{3 \uparrow, 0 \downarrow\}$ & -2.971 & -2.985 & -3.001 & -47.4 & -47.6 & -47.8 & 52.7 & 56.6 & 57.3 \\
\hline $\mathrm{O}$ & $2 s^{2} 2 p^{4}\{3 \uparrow, 1 \downarrow\}$ & -2.830 & -2.849 & -2.853 & 127.0 & 127.8 & 128.0 & -151.3 & -158.0 & -159.8 \\
\hline $\mathrm{F}$ & $2 s^{2} 2 p^{5}\{3 \uparrow, 2 \downarrow\}$ & -1.533 & -1.546 & -1.550 & -954.8 & -962.9 & -965.4 & 1237.4 & 1257.7 & 1261.5 \\
\hline $\mathrm{Sc}$ & $3 d^{1}\{1 \uparrow, 0 \downarrow\} 4 s^{2}$ & -0.581 & -0.588 & -0.610 & -93.5 & -94.7 & -98.2 & 78.7 & 72.5 & 75.5 \\
\hline $\mathrm{Ti}$ & $3 d^{2}\{2 \uparrow, 0 \downarrow\} 4 s^{2}$ & -1.131 & -1.145 & -1.168 & 21.1 & 21.4 & 21.8 & -12.8 & -11.7 & -10.7 \\
\hline $\mathrm{V}$ & $3 d^{3}\{3 \uparrow, 0 \downarrow\} 4 s^{2}$ & -2.505 & -2.563 & -2.637 & -145.6 & -149.0 & -153.3 & 108.4 & 98.5 & 100.6 \\
\hline $\mathrm{Mn}$ & $3 d^{5}\{5 \uparrow, 0 \downarrow\} 4 s^{2}$ & -3.817 & -3.906 & -4.090 & -124.9 & -127.8 & -133.8 & 81.3 & 69.4 & 70.8 \\
\hline $\mathrm{Cu}$ & $3 d^{10} 4 s^{1}\{1 \uparrow, 0 \downarrow\}$ & -0.241 & -0.246 & -0.250 & -42.3 & -43.2 & -43.9 & 6044.7 & 5984.3 & 5979.0 \\
\hline $\mathrm{Zn}$ & $3 d^{10} 4 s^{2}$ & 0.0 & 0.0 & 0.0 & 0.0 & 0.0 & 0.0 & 0.0 & 0.0 & 0.0 \\
\hline
\end{tabular}

aSDA (Ref. 31) exchange-correlation functional.

${ }^{\text {b} G G A ~(R e f . ~ 29) ~ e x c h a n g e-c o r r e l a t i o n ~ f u n c t i o n a l . ~}$ 


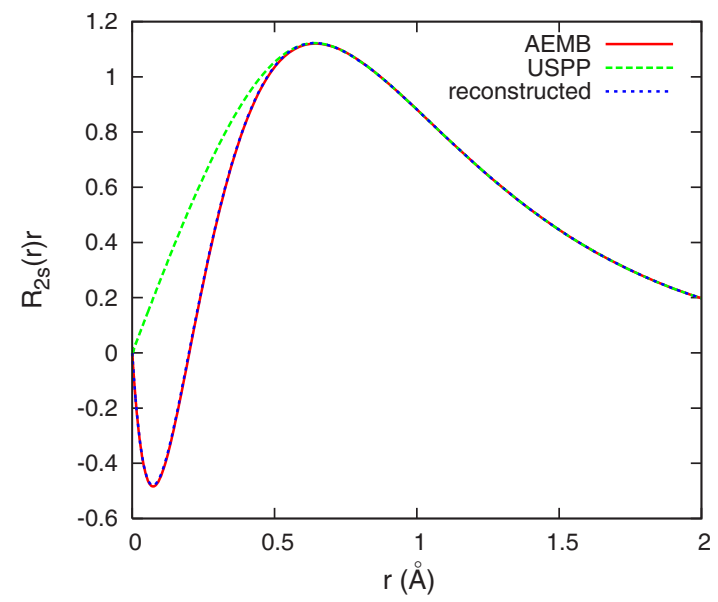

FIG. 2. (Color online) Radial part of $2 s$ orbital, $R_{2 s}(r) r$, obtained directly from an all-electron mixed-basis calculation [AEMB (solid line)], as obtained from reconstructing the pseudo-wave-functions (dotted line) and as obtained from corresponding ultrasoft pseudopotential calculation [USPP (dashed line)].

For both $\rho_{s}^{c}(\mathbf{0})$ and $A_{i s o}^{c}$, the table reveals that both LSDA $^{31}$ and $\mathrm{GGA}^{29}$ functionals give comparable results: for most atoms $1 \%$ or less, and about $2 \%$ for $\mathrm{V}$ and $\mathrm{Mn}$. It is also apparent that there is excellent agreement between our PCLP and the AEMB methods: for first-row elements, the difference is about $1 \%$ (both LSDA); for $3 d$ transition metals also, in spite of the different character of the SOMO, good agreement between PLCP and AEMB exists with the largest differences occurring when the number of unpaired $3 d$ electrons is largest. Even in the extreme case of $\mathrm{Mn}$, with the maximum number of unpaired electrons, the difference between PLCP and AEMB is still only 7\% (LSDA).

On the other hand, the contribution of valence levels to the isotropic HFP turns out to be more sensitive to the choice of exchange-correlation functional as also recently reported by Declerck et al. ${ }^{21}$ According to Table I, for first-row elements, the difference between the LSDA and GGA results of $A_{i s o}^{v}$ varies from $2 \%$ (for C) to $7 \%$ (for N). Such a difference becomes even more pronounced for $3 d$ transition metal atoms. In the extreme case of Mn, the LSDA value of $A_{i s o}^{v}$ differs about $17 \%$ from the corresponding GGA value. The comparison between PCLP and AEMB results (both LSDA) reveals a comparable difference for first-row elements, while a better agreement between the two sets of results can be achieved for $3 d$ transition metal elements. An interesting observation is that the agreement between GGA PCLP and LSDA AEMB results is systematically better than that between LSDA PCLP and the LSDA AEMB results.

Both PLCP and AEMB show that the value of $\rho_{s}^{c}(\mathbf{0})$ increases with the number of unpaired electrons. For $\mathrm{Zn}$, with fully occupied valence orbitals $\left(3 d^{10} 4 s^{2}\right), \rho_{s}^{c}(\mathbf{0})$ becomes zero, whereas for $\mathrm{Mn}\left(3 d^{5} 4 s^{2}\right)$, the absolute value of $\rho_{s}^{c}(\mathbf{0})$ is the largest among the elements considered here. Furthermore, Table I shows that $\rho_{s}^{c}(0)$ for elements with non-s-like SOMO (e.g., F or Sc) is considerably larger than that for $s$-like SOMO (e.g., Cu). These are all in accordance with our earlier assumptions that the strength of induced core spin polarization depends sensitively on the number and type of unpaired electrons in SOMOs [see Eq. (17)]. It is to be noted that $\rho_{s}^{c}(\mathbf{0})$ is always negative. ${ }^{8-10}$ For the first-row elements, it can be explained as follows: according to the Pauli exclusion principle, the exchange interaction induced by unpaired electrons is attractive but applicable to electrons in the same spin channel only. ${ }^{8-10,37}$ As a result, the core $1 s$ electrons of spin majority type are pulled a little outward, thereby leaving behind a slight depletion of their corresponding charge density at the vicinity of nucleus. Thus, the spin density associated with core electrons becomes negative. For $3 d$ transition metals, a more complicated mechanism is needed for a proper interpretation of the negative sign of the core spin densities (see our discussion in Sec. IV B of Ref. 14).

The hyperfine calculations of molecular radicals and transition metal complexes provide a more practically relevant test than atomic calculations. Table II shows the isotropic HFPs as computed with our method, the recently proposed CSPC method, ${ }^{20}$ and the AEMB method, as well as the experimental data, for a series of molecular radicals containing first-row elements. At all the nuclei in each of the molecules, our results are similar to the CSPC results and are also in good agreement with the AEMB and the experimental results. Obviously, there is no contribution from core levels to $A_{\text {iso }}$ of ${ }^{1} \mathrm{H}$. For the other atoms, the comparison between the contributions of core and valence levels to the isotropic HFPs reveals that the former are always lower in magnitude and with an opposing sign as compared to the corresponding valence contributions. A more detailed comparison of the two contributions allows us to divide such molecules, on the basis of their SOMO type, into two main groups.

The first group, including $\mathrm{CH}_{3}, \mathrm{C}_{3} \mathrm{H}_{5}$, and $\mathrm{H}_{2} \mathrm{CN}$, is characterized by an unpaired electron occupying a pure $p(\pi)$-type orbital. The characteristic feature of this group of molecules is that the contributions of both core and valence levels to $A_{\text {iso }}$ are relatively moderate and comparable to each other. In the second group, including $\mathrm{C}_{2} \mathrm{H}_{3}, \mathrm{HCO}, \mathrm{FCO}$, and $\mathrm{NO}_{2}$, the unpaired electron occupies an $s p$-hybridized $\sigma$ orbital. This implies that the $s$ character of SOMO contributes directly to the spin density at the nuclei of the radical. As a result, $A_{\text {iso }}$ of these species is substantially dominated by valence orbitals with large values, as shown in Table II.

To further clarify the above classification, a sample from each group, $\mathrm{C}_{3} \mathrm{H}_{5}$ and $\mathrm{HCO}$, is analyzed in more detail. In $\mathrm{C}_{3} \mathrm{H}_{5}$, all the $\sigma$ molecular orbitals (MOs) are doubly occupied. Thus, the unpaired electron can only be distributed among three $\pi$ MOs where each $\pi$ MO is localized on one of the carbon atoms. According to Table II, the distribution of $\rho^{S O M O}$ is such that the sign of the spin density at the position of the central carbon atom, ${ }^{13} \mathrm{C}^{\alpha}$, becomes negative while it is positive for the two outer carbon atoms, ${ }^{13} \mathrm{C}^{\beta}$. The simplest configuration that can be used to describe such a distribution for $\rho^{S O M O}$ is ${ }^{47}$

$$
\rho^{\text {SOMO }}=\frac{2}{3}\left|\phi_{\pi \beta}^{L}\right|^{2}-\frac{1}{3}\left|\phi_{\pi \alpha}\right|^{2}+\frac{2}{3}\left|\phi_{\pi \beta}^{R}\right|^{2},
$$

where the superscripts $R$ and $L$ indicate the left and the right ${ }^{13} \mathrm{C}^{\beta}$ atoms, respectively. This implies that the induced spin 
TABLE II. Comparison of isotropic HFP for a number of molecular radicals (in MHz). The values labeled "PCLP" were computed with Eq. (1) using spin densities obtained from Eqs. (17) and (11) for core and valence contributions, respectively, "CSPC" refers to core spin-polarization correction method results from Ref. 20, "AEMB" refers to all-electron mixed-basis calculations, and "Expt." refers to experimental results reported in Refs. 37-46.

\begin{tabular}{|c|c|c|c|c|c|c|c|c|c|}
\hline \multirow{4}{*}{$\begin{array}{l}\text { Molecule } \\
\mathrm{CH}_{3}\end{array}$} & \multirow{4}{*}{$\frac{\text { Lewis structure }}{\dot{\mathrm{C}} \mathrm{H}_{3}}$} & \multirow{3}{*}{$\frac{\text { Nucleus }}{{ }^{1} \mathrm{H}}$} & \multicolumn{3}{|c|}{ PCLP } & \multirow{3}{*}{$\frac{\text { CSPC }}{-65.3}$} & \multirow{3}{*}{$\begin{array}{c}\text { AEMB } \\
-69.8\end{array}$} & \multirow{2}{*}{\multicolumn{2}{|c|}{ Expt. }} \\
\hline & & & \multirow[t]{2}{*}{ Core } & \multirow{2}{*}{$\begin{array}{c}\text { Valence } \\
-65.6\end{array}$} & \multirow{2}{*}{$\begin{array}{c}\text { Total } \\
-65.6\end{array}$} & & & & \\
\hline & & & & & & & & $-70.3^{\mathrm{a}}$ & $-64.5^{b}$ \\
\hline & & ${ }^{13} \mathrm{C}$ & -110.1 & 186.4 & 76.3 & 74.3 & 82.7 & $79.6^{\mathrm{a}}$ & $107.3^{\mathrm{b}}$ \\
\hline $\mathrm{C}_{2} \mathrm{H}_{3}$ & $(\mathrm{H} \dot{\mathrm{C}})^{\alpha}=\left(\mathrm{CH}_{2}\right)^{\beta}$ & ${ }^{1} \mathrm{H}^{\alpha}$ & & 40.4 & 40.4 & 43.7 & 39.8 & & 35.9 \\
\hline & & ${ }^{1} \mathrm{H}_{\alpha}^{\beta}$ & & 192.5 & 192.5 & 181.9 & 193.6 & & 184.7 \\
\hline & & ${ }^{1} \mathrm{H}_{s}^{\beta}$ & & 112.1 & 112.1 & 113.8 & 115.2 & & 111.0 \\
\hline & & ${ }^{13} \mathrm{C}^{\alpha}$ & -104.8 & 404.4 & 299.6 & 297.1 & 303.2 & & 301.5 \\
\hline & & ${ }^{13} C^{\beta}$ & 10.1 & -26.3 & -16.2 & -11.5 & -22.4 & & -24.1 \\
\hline $\mathrm{C}_{3} \mathrm{H}_{5}$ & $\left(\mathrm{H}_{2} \mathrm{C}\right)^{\beta \cdots}(\mathrm{CH})^{\alpha \cdots}\left(\mathrm{CH}_{2}\right)^{\beta}$ & ${ }^{1} \mathrm{H}^{\alpha}$ & & 10.1 & 10.1 & 10.6 & 12.0 & & 11.5 \\
\hline & & ${ }^{1} \mathrm{H}^{\beta 1}$ & & -41.5 & -41.5 & -42.0 & -41.8 & & -41.5 \\
\hline & & ${ }^{1} \mathrm{H}^{\beta 2}$ & & -38.4 & -38.4 & -38.9 & -37.8 & & -38.9 \\
\hline & & ${ }^{13} \mathrm{C}^{\alpha}$ & 18.2 & -63.1 & -44.9 & -40.9 & -44.8 & & -48.2 \\
\hline & & ${ }^{13} C^{\beta}$ & -68.1 & 113.5 & 45.4 & 47.6 & 50.2 & & 61.4 \\
\hline $\mathrm{H}_{2} \mathrm{CN}$ & $\mathrm{H}_{2} \mathrm{C}=\dot{\mathrm{N}}$ & ${ }^{1} \mathrm{H}$ & & 236.8 & 236.8 & 238.8 & 237.1 & & 233.2 \\
\hline & & ${ }^{13} \mathrm{C}$ & 13.7 & -79.9 & -66.2 & -61.9 & -70.6 & & -81.0 \\
\hline & & ${ }^{14} \mathrm{~N}$ & -52.7 & 67.0 & 14.3 & 11.8 & 21.3 & & 26.1 \\
\hline $\mathrm{HCO}$ & $\mathrm{H}-\dot{\mathrm{C}}=\mathrm{O}$ & ${ }^{1} \mathrm{H}$ & & 375.8 & 375.8 & 375.0 & 378.3 & & 379.5 \\
\hline & & ${ }^{13} \mathrm{C}$ & -63.0 & 453.7 & 390.7 & 411.1 & 379.7 & & 375.2 \\
\hline & & ${ }^{17} \mathrm{O}$ & 35.0 & -66.4 & -31.4 & -26.6 & -32.8 & & -42.3 \\
\hline FCO & $\mathrm{F}-\dot{\mathrm{C}}=\mathrm{O}$ & ${ }^{19} \mathrm{~F}$ & -184.4 & 1109.5 & 925.1 & 972.2 & 882.5 & & 906.0 \\
\hline & & ${ }^{13} \mathrm{C}$ & -42.9 & 852.5 & 809.6 & 822.8 & 793.4 & & 803.2 \\
\hline & & ${ }^{17} \mathrm{O}$ & 33.9 & -76.3 & -42.4 & -46.5 & -44.8 & & \\
\hline $\mathrm{NO}_{2}$ & $\mathrm{NO}_{2}$ & ${ }^{14} \mathrm{~N}$ & -8.1 & 170.1 & 162.0 & 160.6 & 146.0 & & 153.6 \\
\hline & & ${ }^{17} \mathrm{O}$ & 37.5 & -85.2 & -47.7 & -51.8 & -54.4 & $-45.7 \sim$ & -56.9 \\
\hline
\end{tabular}

axperimental results for a hypothetical nonvibrating molecule from Ref. 37.

birect experimental results from Ref. 38.

polarization of both core $1 s$ and valence $2 s$ orbitals of ${ }^{13} \mathrm{C}^{\alpha}$ must be lower in magnitude with opposite sign compared to that of ${ }^{13} \mathrm{C}^{\beta}$ atoms. Interestingly, the calculated isotropic HFPs confirm the accuracy of this simple model.

For $\mathrm{HCO}$, the unpaired electron, which occupies a $\sigma \mathrm{MO}$, is substantially localized on the $\mathrm{H}-\mathrm{C}$ bond. Our calculations indicate that the $s$ character of $\rho^{S O M O}$ on ${ }^{1} \mathrm{H}$ and ${ }^{13} \mathrm{C}$ is $26 \%$ and $6.9 \%$, respectively. As a result, the $A_{\text {iso }}$ values of the corresponding atoms are significantly dominated by the $s$ character of the SOMO (see Table II). On the other hand, the $p$ character of the SOMO leads to a slight spin polarization of core $1 s$ orbitals, thereby leaving behind a small negative contribution to the isotropic HFP of ${ }^{13} \mathrm{C}$.

Having confirmed the accuracy of our method for firstrow elements and the related molecular radicals, we apply it to the calculation of both isotropic and anisotropic HFPs of a series of $3 d$ transition metal oxides. The results along with those obtained from AEMB calculations and all-electron
Gaussian-type orbital (AE-GTO) calculations by Munzarova and Kaupp ${ }^{48}$ as well as the experimental data are shown in Table III. The overall agreement between our method, the AEMB and AE-GTO results, and experiment appears reasonable. The only exception is the ligand component of $\mathrm{CuO}$ for which both $A_{\text {iso }}$ and $A_{\text {aniso }}$ obtained from our PCLP and AEMB calculations are in better agreement with experiment than the AE-GTO results. A possible explanation for the anomalous AE-GTO results might be a poor basis set selection.

As for the molecular radicals, Table III shows that HFPs depend sensitively on the type of SOMO. In $\mathrm{ScO}, \mathrm{TiO}$, and $\mathrm{VO}$, the $\sigma$-type SOMOs have predominantly metal $4 s$ character with some mixing from $3 d_{z}^{2}$ and $4 p$. This explains the large isotropic HFPs on the metal atoms, while the anisotropy is relatively less pronounced. It is to be noted that $g_{I}$ of ${ }^{47} \mathrm{Ti}$ is negative so that the related HFPs become negative. In going to higher spin multiplicities, $\mathrm{MnO}$ exhibits another 
TABLE III. Hyperfine parameters (in MHz) for transition metal complexes as computed using PCLP, AEMB, and AE-GTO (from Ref. 48) methods, and as experimentally measured (Expt.) (as reported in Ref. 48).

\begin{tabular}{|c|c|c|c|c|c|c|c|c|}
\hline \multirow[b]{2}{*}{ Complex } & \multirow[b]{2}{*}{ Nucleus } & \multirow[b]{2}{*}{ HFP } & \multicolumn{3}{|c|}{ PCLP } & \multirow[b]{2}{*}{ AE-GTO } & \multirow[b]{2}{*}{ AEMB } & \multirow[b]{2}{*}{ Expt. } \\
\hline & & & Core & Valence & Total & & & \\
\hline \multirow[t]{4}{*}{${ }^{45} \mathrm{Sc}{ }^{17} \mathrm{O}$} & ${ }^{45} \mathrm{Sc}$ & $A_{\text {iso }}$ & -67.7 & 2002.7 & 1935.0 & 1933.5 & 1966.1 & 1947.3 \\
\hline & & $A_{\text {aniso }}$ & -4.3 & 39.8 & 35.5 & 17.3 & 32.7 & 24.8 \\
\hline & ${ }^{17} \mathrm{O}$ & $A_{i s o}$ & 0.9 & -19.0 & -18.1 & -19.8 & -23.1 & -20.3 \\
\hline & & $A_{\text {aniso }}$ & & 2.2 & 2.2 & -0.2 & 1.5 & 0.4 \\
\hline \multirow[t]{4}{*}{${ }^{47} \mathrm{Ti}^{17} \mathrm{O}$} & ${ }^{47} \mathrm{Ti}$ & $A_{\text {iso }}$ & 31.3 & -257.0 & -225.7 & -246.3 & -237.3 & -241.0 \\
\hline & & $A_{\text {aniso }}$ & 1.8 & -13.1 & -11.3 & -4.7 & -5.2 & \\
\hline & ${ }^{17} \mathrm{O}$ & $A_{i s o}$ & 1.5 & -17.2 & -15.7 & -7.3 & -20.1 & \\
\hline & & $A_{\text {aniso }}$ & & -6.1 & -6.1 & -1.6 & -3.2 & \\
\hline \multirow[t]{4}{*}{${ }^{51} \mathrm{~V}^{17} \mathrm{O}$} & ${ }^{51} \mathrm{~V}$ & $A_{i s o}$ & -159.8 & 931.1 & 771.3 & 811.9 & 778.9 & 778.0 \\
\hline & & $A_{\text {aniso }}$ & 5.5 & -49.8 & -44.3 & -47.7 & -55.6 & -41.3 \\
\hline & ${ }^{17} \mathrm{O}$ & $A_{i s o}$ & 1.0 & -7.9 & -6.9 & -2.4 & -3.3 & \\
\hline & & $A_{\text {aniso }}$ & & -0.4 & -0.4 & -3.1 & -2.5 & \\
\hline \multirow[t]{4}{*}{${ }^{55} \mathrm{Mn}{ }^{17} \mathrm{O}$} & ${ }^{55} \mathrm{Mn}$ & $A_{\text {iso }}$ & -230.4 & 682.8 & 452.4 & 524.0 & 481.6 & 479.9 \\
\hline & & $A_{\text {aniso }}$ & 7.6 & -43.2 & -35.6 & -24.3 & -28.7 & -16.1 \\
\hline & ${ }^{17} \mathrm{O}$ & $A_{i s o}$ & 2.4 & -16.6 & -14.2 & -5.3 & -6.1 & \\
\hline & & $A_{\text {aniso }}$ & & 15.8 & 15.8 & 8.3 & 9.5 & \\
\hline \multirow[t]{4}{*}{${ }^{63} \mathrm{Cu}{ }^{17} \mathrm{O}$} & ${ }^{63} \mathrm{Cu}$ & $A_{\text {iso }}$ & -778.3 & 309.2 & -469.1 & -678.1 & -493.9 & -483.6 \\
\hline & & $A_{\text {aniso }}$ & -11.3 & 49.8 & 38.5 & 41.8 & 34.0 & 24.1 \\
\hline & ${ }^{17} \mathrm{O}$ & $A_{i s o}$ & 10.7 & -52.1 & -41.4 & -5.2 & -38.6 & \\
\hline & & $A_{\text {aniso }}$ & & 49.8 & 49.8 & -112.6 & 57.4 & \\
\hline
\end{tabular}

a Using DFT-BPW91 (Ref. 48).

type of SOMOs. Comparing with the previous cases, $\mathrm{MnO}$ has two additional SOMOs, antibonding orbitals with metal $3 d_{\pi}$ and $4 p_{\pi}$ as well as ligands with $2 p_{\pi}$ character. Due to the large number of $d$-type SOMOs, spin-polarization effects via the core shells are more pronounced. Suffice it to say that the contribution of the core levels $(-230 \mathrm{MHz})$ to the total value of the isotropic $\mathrm{HFP}$ of ${ }^{55} \mathrm{Mn}(+452.4 \mathrm{MHz})$ is very significant. Thus, the neglect of core spin polarization definitely leads to a large error in the isotropic HFP. Finally, in $\mathrm{CuO}$, the situation becomes even more critical. Here, there is no contribution from the metal $4 s$ states to the SOMO. Instead, the SOMO is due to $3 d$-type orbitals and the negative core contribution is actually larger than that of the valence states.

Since for most of the present transition metal oxides, $\rho^{\text {SOMO }}$ is significantly localized on the metal atoms, ${ }^{49}$ both $A_{\text {iso }}$ and $A_{\text {aniso }}$ of the ligand part turn out to be small. The only exception is $\mathrm{CuO}$, whose spin density is slightly delocalized on the ${ }^{17} \mathrm{O}$ atom. ${ }^{48}$

As a final verification of the PLCP method, we have computed the HFPs pertaining to a point defect in a II-VI crystal i.e., a positively charged $\mathrm{Zn}$ interstitial $\left(\mathrm{Zn}_{i}^{+}\right)$in $\mathrm{ZnSe}$. A 32 +1 atom cell was constructed with $16 \mathrm{Zn}$ and $16 \mathrm{Se}$ on the cubic zinc-blende $\mathrm{ZnSe}$ sites where the cation, $\mathrm{Zn}^{+}$, occupies a tetrahedral interstitial site surrounded by Se atoms $\left(T_{d}^{\mathrm{Se}}\right){ }^{51}$ The $\mathrm{Zn}$ interstitial caused relaxations: in our calculations the first (second) neighbor shells $\mathrm{Se}_{1 \mathrm{NN}}\left(\mathrm{Zn}_{2 \mathrm{NN}}\right)$ move outward by $0.12 \AA(0.07 \AA)$ along $\langle 111\rangle(\langle 100\rangle)$ directions, similar to relaxations found by van de Walle and Blöchl. ${ }^{15}$ The calculated HFPs for the corresponding $\mathrm{Zn}_{i}^{+}$and its first $\left(\mathrm{Se}_{1 \mathrm{NN}}\right)$, second $\left(\mathrm{Zn}_{2 \mathrm{NN}}\right)$, and third $\left(\mathrm{Se}_{3 \mathrm{NN}}\right)$ are listed in Table IV, and compared with theoretical ${ }^{15}$ and experimental ${ }^{50}$ data. The table indicates that the HFPs obtained by PCLP are in good agreement with the experimental data; in fact, the agreement is a little better than the previous calculation. ${ }^{15}$ Our calculations show that the spin density is substantially localized on the $\mathrm{Zn}_{i}^{+}$and its first neighbors, $\mathrm{Se}_{1 \mathrm{NN}}$, and is significantly $4 s$-like. In the case of $\mathrm{Se}_{1 \mathrm{NN}}$, it has an additional minor contribution from its valence $4 p$ orbitals. Therefore, the core contribution is rather significant on $\mathrm{Se}_{1 \mathrm{NN}}$, and it explains why the earlier calculation without core contribution ${ }^{15}$ overestimated the HFP on this site.

\section{CONCLUSIONS}

A computationally expedient, density-functional-theorybased method for calculating hyperfine parameters within pseudopotential formalism has been presented. The accurate 
TABLE IV. Hyperfine parameters (in MHz) for $\mathrm{Zn}_{i}^{+}$at the $T_{d}^{\mathrm{Se}}$ site in $\mathrm{ZnSe}$. Results of this work [previous pseudopotential calculation (Ref. 15)] are listed in columns under PCLP (PP). Values are given for the interstitial at the center of the defect as well as for the first $\left({ }^{77} \mathrm{Se}\right)$, second $\left({ }^{67} \mathrm{Zn}\right)$, and third $\left({ }^{77} \mathrm{Se}\right)$ neighbors.

\begin{tabular}{lcccccc}
\hline \hline & & \multicolumn{5}{c}{ PCLP } \\
\cline { 3 - 5 } Nucleus & HFP & Core & Valence & Total & PP & Expt. ${ }^{\mathrm{b}}$ \\
\hline${ }^{67} \mathrm{Zn}_{i}^{+}$ & $A_{\text {iso }}$ & -11.1 & 1093.1 & 1082.0 & 1078 & 1089 \\
& $A_{\text {aniso }}$ & -0.1 & 5.2 & 5.1 & & \\
${ }^{77} \mathrm{Se}_{1 \mathrm{NN}}$ & $A_{\text {iso }}$ & -84.3 & 693.5 & 609.2 & 736 & 481 \\
& $A_{\text {aniso }}$ & -0.9 & 15.2 & 14.3 & 11 & 16.8 \\
${ }^{67} \mathrm{Zn}_{2 \mathrm{NN}}$ & $A_{\text {iso }}$ & 0.0 & 10.7 & 10.7 & 10 & \\
& $A_{\text {aniso }}$ & 0.0 & 0.0 & 0.0 & & \\
${ }^{77} \mathrm{Se}_{3 \mathrm{NN}}$ & $A_{\text {iso }}$ & -0.1 & 41.9 & 41.8 & 55 & 37.5 \\
& $A_{\text {aniso }}$ & 0.0 & 0.6 & 0.6 & & \\
\hline \hline
\end{tabular}

Reference 15.

${ }^{\mathrm{b}}$ Reference 50 as cited in Ref. 15.

representation of the spin density at and in the vicinity of the nucleus was achieved by reconstructing the full-nodal behavior of the wave functions with inclusion of core spin polarization via first-order perturbation theory. The method was successfully applied to calculate the hyperfine parameters of various molecular radicals, complexes, and a crystalline point defect containing first-row of elements and $3 d$ transition metals. The current method is highly versatile because it does not impose restrictions on the exchange-correlation functional in the pseudopotential calculations. The additional information regarding the code with which these calculations were carried out can be obtained upon request from Bahramy.

\section{ACKNOWLEDGMENT}

The authors gratefully acknowledge the Center for Computational Materials Science at the Institute for Materials Research for allocations on the Hitachi SR8000 supercomputer system. *saeed@imr.edu

${ }^{1}$ A. Abragam and B. Bleaney, Electronic Paramagnetic Resonance of Transition Ions (Clarendon, Oxford, 1970).

${ }^{2}$ J. A. Weil, J. R. Bolton, and J. E. Wertz, Electron Paramagnetic Resonance: Elementary Theory and Practical Applications (Wiley, New York, 1994).

${ }^{3}$ I. Bertini, C. Luchinat, and G. Parigi, Solution NMR of Paramagnetic Molecules (Elsevier, Amsterdam, 2001).

${ }^{4}$ D. Feller and E. R. Davidson, Theor. Chim. Acta 68, 57 (1985).

${ }^{5}$ S. A. Perera, J. D. Watts, and R. J. Bartlett, J. Chem. Phys. 100, 1425 (1994).

${ }^{6}$ R. K. Nesbet, Proc. R. Soc. London, Ser. A 230, 312 (1955).

${ }^{7}$ G. W. Pratt, Jr., Phys. Rev. 102, 1303 (1956).

${ }^{8}$ A. J. Freeman and R. E. Watson, Phys. Rev. Lett. 5, 498 (1960).

${ }^{9}$ R. E. Watson and A. J. Freeman, Phys. Rev. 123, 2027 (1961).

${ }^{10}$ R. E. Watson and A. J. Freeman, Phys. Rev. Lett. 6, 277 (1961).

${ }^{11}$ P. Hohenberg and W. Kohn, Phys. Rev. 136, B864 (1964).

${ }^{12}$ H. Overhof, M. Scheffler, and C. M. Weinert, Phys. Rev. B 43, 12494 (1991).

${ }^{13}$ H. Katayama-Yoshida and N. Hamada, Phys. Rev. B 35, 407 (1987).

${ }^{14}$ M. S. Bahramy, M. H. F. Sluiter, and Y. Kawazoe, Phys. Rev. B 73, 045111 (2006).
${ }^{15}$ C. G. Van de Walle and P. E. Blöchl, Phys. Rev. B 47, 4244 (1993).

${ }^{16}$ J. Vackář, M. Hyt'ha, and A. Šimůnek, Phys. Rev. B 58, 12712 (1998).

${ }^{17}$ B. Meyer, K. Hummler, C. Elsässer, and M. Fähnle, J. Phys.: Condens. Matter 7, 9201 (1995).

${ }^{18}$ B. Hetényi, F. D. Angelis, P. Giannozzi, and R. Car, J. Chem. Phys. 115, 5791 (2001).

${ }^{19}$ P. E. Blöchl, Phys. Rev. B 50, 17953 (1994).

${ }^{20}$ O. V. Yazyev, I. Tavernelli, L. Helm, and U. Röthlisberger, Phys. Rev. B 71, 115110 (2005).

${ }^{21}$ R. Declerck, E. Pauwels, V. Van Speybroeck, and M. Waroquier, Phys. Rev. B 74, 245103 (2006).

${ }^{22}$ D. A. Goodings, Phys. Rev. 123, 1706 (1961).

${ }^{23}$ G. D. Gaspari, W.-M. Shyu, and T. P. Das, Phys. Rev. 134, A852 (1964).

${ }^{24}$ D. R. Lide, Handbook of Chemistry and Physics, 85th ed. (CRC, Boca Raton, 2004), pp. 9-93.

${ }^{25}$ F. Herman and S. Skillman, Atomic Structure Calculations (Prentice-Hall, Englewood Cliffs, NJ, 1963).

${ }^{26}$ G. L. Oliver and J. P. Perdew, Phys. Rev. A 20, 397 (1979).

${ }^{27}$ T. Asada and K. Terakura, Phys. Rev. B 46, 13599 (1992).

${ }^{28}$ M. Battocletti, H. Ebert, and H. Akai, Phys. Rev. B 53, 9776 
(1996).

${ }^{29}$ J. P. Perdew, Electronic Structure of Solids '91, edited by P. Ziesche and H. Eschrig (Akademie Verlag, Berlin, 1991), p. 11; J. P. Perdew and Y. Wang, Phys. Rev. B 45, 13244 (1992).

${ }^{30}$ J. P. Perdew, J. A. Chevary, S. H. Vosko, K. A. Jackson, M. R. Pederson, D. J. Singh, and C. Fiolhais, Phys. Rev. B 46, 6671 (1992); 48, 4978(E) (1993).

${ }^{31}$ J. P. Perdew and A. Zunger, Phys. Rev. B 23, 5048 (1981).

${ }^{32}$ D. Vanderbilt, Phys. Rev. B 41, 7892 (1990).

${ }^{33}$ Vienna $a b$ initio software package (VASP), Version 4.6.12, http:// cms.mpi.univie.ac.at/vasp/

${ }^{34}$ R. Pis Diez, computer code ATOMDEF, Version 1.4, 2003, can be downloaded from http://www.quimica.unlp.edu.ar/cequinor/ rpd_en.htm

${ }^{35}$ J. P. Perdew, K. Burke, and M. Ernzerhof, Phys. Rev. Lett. 77, 3865 (1996).

${ }^{36}$ S. H. Vosko, L. Wilk, and M. Nusair, Can. J. Phys. 58, 1200 (1980).

${ }^{37}$ D. M. Chipman, Theor. Chim. Acta 82, 93 (1992).

${ }^{38}$ E. Hirota and C. Yamada, J. Mol. Spectrosc. 96, 175 (1985).

${ }^{39}$ R. W. Fessenden and R. H. Schuler, J. Chem. Phys. 39, 2147 (1963).

${ }^{40}$ R. W. Fessenden, J. Phys. Chem. 71, 74 (1967).
${ }^{41}$ H. J. McManus, R. W. Fessenden, and D. M. Chipman, J. Phys. Chem. 92, 3778 (1988).

${ }^{42}$ H. J. McManus, R. W. Fessenden, and D. M. Chipman, J. Phys. Chem. 92, 3781 (1988).

${ }^{43}$ F. J. Adrian, B. F. Kim, and J. Bohandy, J. Chem. Phys. 82, 1804 (1985).

${ }^{44}$ F. J. Adrian, E. L. Cochran, and V. A. Bowers, J. Chem. Phys. 43, 462 (1965).

${ }^{45}$ E. L. Cochran, F. J. Adrian, and V. A. Bowers, J. Chem. Phys. 44, 4626 (1966).

${ }^{46}$ Z. Luz, A. Reuveni, R. W. Holmberg, and B. L. Silver, J. Chem. Phys. 51, 4017 (1969).

${ }^{47}$ H. M. McConnell, J. Chem. Phys. 28, 1188 (1958).

${ }^{48}$ M. L. Munzarová and M. Kaupp, J. Phys. Chem. A 103, 9966 (1999).

${ }^{49}$ M. L. Munzarová, P. Kubáček, and M. Kaupp, J. Am. Chem. Soc. 122, 11900 (2000).

${ }^{50}$ F. Rong and G. D. Watkins, Phys. Rev. Lett. 58, 1486 (1987).

${ }^{51}$ The supercell without interstitial has as translation vectors $a_{\mathrm{ZnSe}}(-1,1,1), a_{\mathrm{ZnSe}}(1,-1,1)$, and $a_{\mathrm{ZnSe}}(1,1,-1)$, where $a_{\mathrm{ZnSe}}$ is the lattice parameter of the cubic parent structure. A $6 \times 6$ $\times 6$ Monkhorst-Pack mesh has been used to sample the irreducible Brillouin zone. 